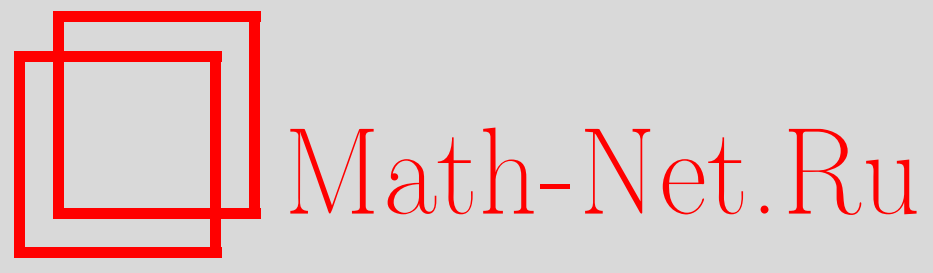

В. А. Гущина, Нелокальная задача А. А. Дезина Для уравнения смешанного эллиптико-гиперболического типа, Вестн. Сам. гос. техн. ун-та. Сер. Физ.-мат. науки, 2016, номер 1, 22-32

DOI: https://doi.org/10.14498/vsgtu1470

Использование Общероссийского математического портала MathNet.Ru подразумевает, что вы прочитали и согласны с пользовательским соглашением

http: //www . mathnet.ru/rus/agreement

Параметры загрузки:

IP : 54.198 .55 .26

26 апреля 2023 г., 14:02:26

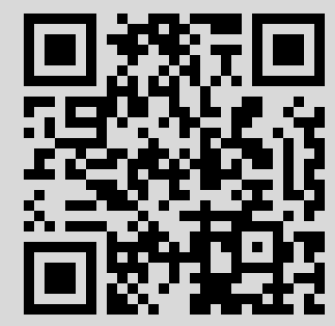


Вестн. Сам. гос. техн. ун-та. Сер. Физ.-мат. науки. 2016. Т. 20, № 1 . С. $22-32$

ISSN: 2310-7081 (online), 1991-8615 (print)

doi: http://dx.doi.org/10.14498/vsgtu1470

УДК 517.957

\title{
НЕЛОКАЛЬНАЯ ЗАДАЧА А. А. ДЕЗИНА ДЛЯ УРАВНЕНИЯ СМЕШАННОГО ЭЛЛИПТИКО-ГИПЕРБОЛИЧЕСКОГО ТИПА
}

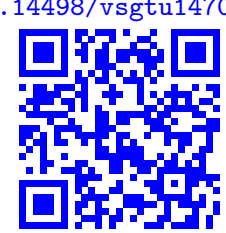

\section{В. А. Гущина}

Самарский государственный социально-педагогический университет,

Россия, 443099, Самара, ул. М. Горького, 65/67.

\begin{abstract}
Аннотация
В данной работе для уравнения смешанного эллиптико-гиперболического типа в прямоугольной области изучена задача с условиями периодичности и нелокальным условием А. А. Дезина. Установлен критерий единственности. Решение задачи построено в виде суммы ортогонального ряда по собственным функциям соответствующей одномерной спектральной задачи. При обосновании сходимости ряда возникает проблема малых знаменателей. В связи с этим установлена оценка отделенности от нуля малых знаменателей с соответствующей асимптотикой. Эта оценка позволила при некоторых условиях относительно заданных параметров задачи и функций доказать сходимость построенного ряда в классе регулярных решений и устойчивость решения.
\end{abstract}

Ключевые слова: уравнение смешанного типа, нелокальная задача, критерий единственности, существование, ряд, малые знаменатели, устойчивость.

1. Постановка задачи. Рассмотрим неоднородное уравнение смешанного типа

$$
L u \equiv u_{x x}+(\operatorname{sgn} y) u_{y y}-b^{2} u=F(x, y)
$$

в прямоугольной области

$$
D=\{(x, y) \mid 0<x<l,-\alpha<y<\beta\}
$$

где $l, b, \alpha$ и $\beta$-заданные положительные постоянные, и поставим задачу А. А. Дезина [1].

ЗАДАЧА ДЕзИНА Найти в области $D$ функиию $u(x, y)$, удовлетворяюшую следующим условиям:

$$
\begin{gathered}
u \in C^{1}(\bar{D}) \cap C^{2}\left(D_{-} \cup D_{+}\right) ; \\
L u=F(x, y), \quad(x, y) \in D_{-} \cup D_{+} ;
\end{gathered}
$$

(C) 2016 Самарский государственный технический университет.

\section{Образец для цитирования}

Гущи н а В. А. Нелокальная задача А. А. Дезина для уравнения смешанного эллиптикогиперболического типа // Вестн. Сам. гос. техн. ун-та. Сер. Физ.-мат. науки, 2016. Т. 20, № 1. C. 22-32. doi: 10.14498/vsgtu1470.

\section{Сведения об авторе}

Виолетта Александровна Гущина (violetta.novikova.1991@mail.ru), аспирант, каф. физики, математики и методики обучения. 


$$
\begin{gathered}
u(0, y)-u(l, y)=0, \quad u_{x}(0, y)-u_{x}(l, y)=0, \quad-\alpha \leqslant y \leqslant \beta \\
u(x, \beta)=\varphi(x), \quad 0 \leqslant x \leqslant l ; \\
u_{y}(x,-\alpha)-\lambda u(x, 0)=\psi(x), \quad 0 \leqslant x \leqslant l
\end{gathered}
$$

где $\varphi(x), \psi(x)$ - заданнье достаточно гладкие функиии; $D_{+}=D \cap\{y>0\}$, $D_{-}=D \cap\{y<0\} ; \lambda-$ заданный действительный параметр.

А. А. Дезин в своих работах $[1,2]$ отметил, что метод поиска разрешимых расширений для дифференциальных операторов может быть адаптирован к оператору Лаврентьева-Бицадзе с условиями периодичности по переменной $x$. В работах 3. А. Нахушевой [3], [4, с. 143-153] задача (2)-(6) изучена, когда $\alpha=l, \varphi(x)=\psi(x)=0, b=0, \lambda \geqslant 0, F(x, y)=f(x, y) \cdot H(y)$, где $H(y)-$ функция Хевисайда. Показано, что при $\lambda<0$ однородная задача (т. е. когда $f(x, y) \equiv 0)$ имеет нетривиальные решения.

В работе [5] была изучена задача (2)-(6) при $F(x, y) \equiv 0, b=0$, где установлен критерий единственности решения поставленной задачи. Решение построено в виде суммы ряда по собственным функциям соответствующей одномерной спектральной задачи. При обосновании сходимости ряда возникла проблема малых знаменателей относительно отношения сторон $\alpha / l$ прямоугольника $D_{-}$. При некоторых условиях относительно $\alpha / l, \lambda, \beta$ и функций $\varphi(x), \psi(x)$ показано, что сумма построенного ряда принадлежит классу (2). Доказана также устойчивость решения задачи от заданных функций $\varphi(x)$ и $\psi(x)$.

В данной работе задача (2)-(6) изучена для уравнения $(1)$ при $F(x, y) \equiv 0$ и всех $b \neq 0$. Также установлен критерий единственности. Решение построено в виде суммы ряда Фурье, равномерная сходимость которого зависит от $\alpha / l$, $\lambda, \beta$ и $b$. Установлены теоремы существования и устойчивости решения.

Отметим, что впервые нелокальные задачи для уравнений смешанного типа были изучены в работах [6-8].

2. Единственность решения задачи. Пусть существует решение задачи (2)(6) при $F(x, y) \equiv 0$. Следуя $[5,9,10]$ введем функции

$$
\begin{gathered}
u_{0}(y)=\frac{1}{\sqrt{l}} \int_{0}^{l} u(x, y) d x, \quad u_{k}(y)=\sqrt{\frac{2}{l}} \int_{0}^{l} u(x, y) \cos \mu_{k} x d x \\
v_{k}(y)=\sqrt{\frac{2}{l}} \int_{0}^{l} u(x, y) \sin \mu_{k} x d x, \quad k \in \mathbb{N}
\end{gathered}
$$

и аналогично этим работам относительно функций (7) и (8) получим дифференциальные уравнения

$$
\begin{gathered}
u_{k}^{\prime \prime}(y)-(\operatorname{sgn} y) \gamma_{k}^{2} u_{k}(y)=0, \quad y \in(\alpha, 0) \cup(0, \beta), \\
u_{0}^{\prime \prime}(y)-(\operatorname{sgn} y) b^{2} u=0, \quad y \in(-\alpha, 0) \cup(0, \beta), \\
v_{k}^{\prime \prime}(y)-(\operatorname{sgn} y) \gamma_{k}^{2} v_{k}(y)=0, \quad y \in(-\alpha, 0) \cup(0, \beta), k \in \mathbb{N},
\end{gathered}
$$

где $\gamma_{k}^{2}=\mu_{k}^{2}+b^{2}$. Найдем общее решение уравнения (9):

$$
u_{k}(y)= \begin{cases}c_{k} e^{\gamma_{k} y}+d_{k} e^{-\gamma_{k} y}, & y>0 \\ a_{k} \cos \gamma_{k} y+b_{k} \sin \gamma_{k} y, & y<0\end{cases}
$$


где $a_{k}, b_{k}, c_{k}$ и $d_{k}$ - произвольные постоянные. Выберем эти постоянные так, чтобы в силу (2) выполнялись условия сопряжения

$$
u_{k}(0+0)=u_{k}(0-0), \quad u_{k}^{\prime}(0+0)=u_{k}^{\prime}(0-0) .
$$

Удовлетворяя функции (12) условиям (13), найдем

$$
c_{k}=\frac{a_{k}+b_{k}}{2}, \quad d_{k}=\frac{a_{k}-b_{k}}{2} .
$$

Тогда с учётом найденных значений $c_{k}$ и $d_{k}$ функции $(12)$ примут вид

$$
u_{k}(y)= \begin{cases}a_{k} \operatorname{ch} \gamma_{k} y+b_{k} \operatorname{sh} \gamma_{k} y, & y>0 \\ a_{k} \cos \gamma_{k} y+b_{k} \sin \gamma_{k} y, & y<0 .\end{cases}
$$

Для нахождения постоянных $a_{k}$ и $b_{k}$ воспользуемся условиями $(5),(6)$ и формулой (7):

$$
\begin{aligned}
& u_{k}(\beta)=\sqrt{\frac{2}{l}} \int_{0}^{l} u(x, \beta) \cos \mu_{k} x d x=\sqrt{\frac{2}{l}} \int_{0}^{l} \varphi(x) \cos \mu_{k} x d x=\varphi_{k} \\
& u_{k}^{\prime}(-\alpha)-\lambda u_{k}(0)=\sqrt{\frac{2}{l}} \int_{0}^{l}\left[u_{y}(x,-\alpha)-\lambda u(x, 0)\right] \cos \mu_{k} x d x= \\
&=\sqrt{\frac{2}{l}} \int_{0}^{l} \psi(x) \cos \mu_{k} x d x=\psi_{k} .
\end{aligned}
$$

Теперь на основании (14)-(16) получим систему

$$
\left\{\begin{array}{l}
a_{k} \operatorname{ch} \gamma_{k} \beta+b_{k} \operatorname{sh} \gamma_{k} \beta=\varphi_{k}, \\
a_{k}\left(\sin \gamma_{k} \alpha-\lambda / \gamma_{k}\right)+b_{k} \cos \gamma_{k} \alpha=\psi_{k} / \gamma_{k}
\end{array}\right.
$$

Если определитель системы (17)

$$
\Delta(k)=\cos \gamma_{k} \alpha \operatorname{ch} \gamma_{k} \beta-\operatorname{sh} \gamma_{k} \beta \sin \gamma_{k} \alpha+\left(\lambda / \gamma_{k}\right) \operatorname{sh} \gamma_{k} \beta \neq 0
$$

при всех $k \in \mathbb{N}$, то данная система имеет единственное решение:

$$
\begin{gathered}
a_{k}=\frac{1}{\Delta(k)}\left(\varphi_{k} \cos \gamma_{k} \alpha-\psi_{k} \frac{\operatorname{sh} \gamma_{k} \beta}{\gamma_{k}}\right), \\
b_{k}=\frac{1}{\Delta(k)}\left(\varphi_{k}\left(\frac{\lambda}{\gamma_{k}}-\sin \gamma_{k} \alpha\right)+\psi_{k} \frac{\operatorname{ch} \gamma_{k} \beta}{\gamma_{k}}\right) .
\end{gathered}
$$

Отметим, что определитель $\Delta(k)$, помимо переменной $k$, зависит также от данных задачи $\alpha, \beta, l, b$ и $\lambda$ как параметров. Подставляя (19) и (20) в (17), найдём окончательный вид функций:

$$
u_{k}(y)= \begin{cases}\varphi_{k} \frac{A_{k}(\alpha, y)}{\Delta(k)}-\psi_{k} \frac{\operatorname{sh} \gamma_{k}(\beta-y)}{\Delta(k)}, & y>0, \\ \varphi_{k} \frac{B_{k}(\alpha, y)}{\Delta(k)}+\psi_{k} \frac{C_{k}(y, \beta)}{\Delta(k)}, & y<0\end{cases}
$$


где

$$
\begin{gathered}
A_{k}(\alpha, y)=\cos \gamma_{k} \alpha \operatorname{ch} \gamma_{k} y-\sin \gamma_{k} \alpha \operatorname{sh} \gamma_{k} y+\left(\lambda / \gamma_{k}\right) \operatorname{sh} \gamma_{k} y \\
B_{k}(\alpha, y)=\cos \gamma_{k}(\alpha+y)+\left(\lambda / \gamma_{k}\right) \sin \gamma_{k} y \\
C_{k}(y, \beta)=\operatorname{ch} \gamma_{k} \beta \sin \gamma_{k} y-\operatorname{sh} \gamma_{k} \beta \cos \gamma_{k} y
\end{gathered}
$$

По аналогичной схеме исходя из уравнения (10) на основании условий сопряжения и граничных условий (5) и (6) найдём

$$
u_{0}(y)= \begin{cases}\varphi_{0} \frac{A_{0}(\alpha, y)}{\Delta(0)}+\psi_{0} \frac{\operatorname{sh} b(\beta-y)}{\Delta(0)}, & y>0, \\ \varphi_{0} \frac{B_{0}(\alpha, y)}{\Delta(0)}+\psi_{0} \frac{C_{0}(y, \beta)}{\Delta(0)}, & y<0\end{cases}
$$

где

$$
\begin{gathered}
\Delta(0)=\operatorname{ch}(b \beta) \cos (b \alpha)-\operatorname{sh}(b \beta) \sin (b \alpha)+(\lambda / b) \operatorname{sh}(b \beta) \neq 0 . \\
A_{0}(\alpha, y)=\cos (b \alpha) \operatorname{ch}(b y)-\sin (b \alpha) \operatorname{sh}(b y)+(\lambda / b) \operatorname{sh}(b y), \\
B_{0}(\alpha, y)=\cos b(\alpha+y)+(\lambda / b) \sin (b y), \\
C_{0}(y, \beta)=\operatorname{ch}(b \beta) \sin (b y)-\operatorname{sh}(b \beta) \cos (b y), \\
\varphi_{0}=\frac{1}{\sqrt{l}} \int_{0}^{l} \varphi(x) d x, \quad \psi_{0}=\frac{1}{\sqrt{l}} \int_{0}^{l} \psi(x) d x .
\end{gathered}
$$

Формулу (25) и условие (26) можно получить исходя из равенств (21)-(24) и (18) при $k=0$.

Повторяя рассуждения, аналогичные при построении формулы (21), на основании общего решения уравнения (11) найдем

$$
v_{k}(y)= \begin{cases}\widetilde{\varphi}_{k} \frac{A_{k}(\alpha, y)}{\Delta(k)}-\widetilde{\psi}_{k} \frac{\operatorname{sh} \gamma_{k}(\beta-y)}{\Delta(k)}, & y>0, \\ \widetilde{\varphi}_{k} \frac{B_{k}(\alpha, y)}{\Delta(k)}+\widetilde{\psi}_{k} \frac{C_{k}(y, \beta)}{\Delta(k)}, & y<0,\end{cases}
$$

где

$$
\widetilde{\varphi}_{k}=\sqrt{\frac{2}{l}} \int_{0}^{l} \varphi(x) \sin \mu_{k} x d x, \quad \widetilde{\psi}_{k}=\sqrt{\frac{2}{l}} \int_{0}^{l} \psi(x) \sin \mu_{k} x d x .
$$

Теперь мы в состоянии доказать теорему единственности решения задачи $(2)-(6)$. Пусть $\varphi(x) \equiv 0, \psi(x) \equiv 0$ и выполнены условия (18) при всех $k \in \mathbb{N}_{0}$. Тогда $\varphi_{k}=\psi_{k}=\widetilde{\varphi}_{k}=\widetilde{\psi}_{k}=0$ при всех $k \in \mathbb{N}_{0}$ и из равенств (21), (25), (27), (7), (8) при всех $y \in[-\alpha, \beta]$ и $k \in \mathbb{N}_{0}$ следует, что

$$
\int_{0}^{1} u(x, y) d x=0, \quad \int_{0}^{l} u(x, y) \cos \mu_{k} x d x=0, \quad \int_{0}^{l} u(x, y) \sin \mu_{k} x d x=0 .
$$

Отсюда в силу полноты системы функций

$$
\left\{\frac{1}{\sqrt{l}}, \sqrt{\frac{2}{l}} \cos \mu_{k} x, \sqrt{\frac{2}{l}} \sin \mu_{k} x\right\}
$$


в пространстве $L_{2}[0, l]$ следует, что $u(x, y)=0$ почти всюду на $[0, l]$ при любом $y \in[-\alpha, \beta]$. Функция $u(x, y) \equiv 0$ в $\bar{D}$, поскольку она непрерывна на $\bar{D}$.

Пусть при некоторых $\alpha, \beta, b, l, \lambda$ и $k=p \in \mathbb{N}_{0}$ нарушено условие (18), т.е. $\Delta(p)=0$. Тогда однородная задача $(2)-(6)($ где $\varphi(x)=\psi(x) \equiv 0)$ имеет нетривиальные решения:

$$
\begin{aligned}
& u_{p}(x, y)=u_{p}(y)\left(A_{1}+A_{2} \cos \gamma_{p} x+A_{3} \sin \gamma_{p} x\right),
\end{aligned}
$$

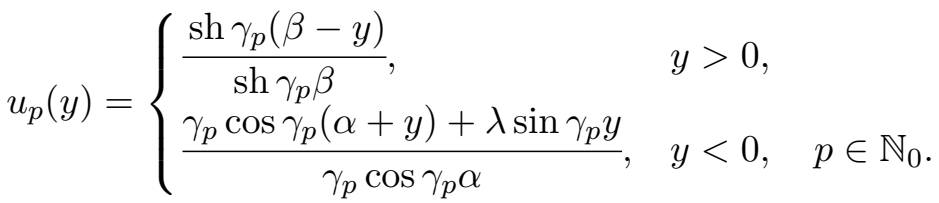

Здесь $A_{1}, A_{2}, A_{3}$ - произвольные постоянные.

Теперь естественно возникает вопрос о существовании корней уравнения $\Delta(p)=0$. Для этого представим его в виде

$$
\Delta(p)=-\sqrt{\operatorname{ch} 2 \gamma_{p} \beta} \sin \left(2 \pi p \widetilde{\alpha} \widetilde{\gamma_{p}}-\theta_{p}\right)+\left(\lambda / \gamma_{p}\right) \operatorname{sh} \gamma_{p} \beta=0
$$

где

$$
\theta_{p}=\arcsin \frac{\operatorname{ch} \gamma_{p} \beta}{\sqrt{\operatorname{ch} 2 \gamma_{p} \beta}}, \quad \widetilde{\alpha}=\frac{\alpha}{l}, \quad \widetilde{\gamma_{p}}=\sqrt{1+(b l / 2 \pi p)^{2}}
$$

Отсюда видно, что уравнение (30) имеет счётное множество нулей относительно

$$
\widetilde{\alpha}=\frac{(-1)^{n}}{2 \pi p \widetilde{\gamma_{p}}} \arcsin \frac{\lambda \operatorname{sh} \gamma_{p} \beta}{\gamma_{p} \sqrt{\operatorname{ch} 2 \gamma_{p} \beta}}+\frac{\theta_{p}}{2 \pi p \widetilde{\gamma_{p}}}+\frac{\pi n}{2 \pi p \widetilde{\gamma_{p}}}, \quad n \in \mathbb{N}_{0}, \quad p \in \mathbb{N},
$$

при условии

$$
\frac{|\lambda|}{\gamma_{p}} \frac{\operatorname{sh} \gamma_{p} \beta}{\sqrt{\operatorname{sh}^{2} \gamma_{p} \beta+\operatorname{ch}^{2} \gamma_{p} \beta}} \leqslant 1
$$

Когда $\gamma_{p} \geqslant|\lambda|$, т.е. $p \geqslant|\lambda| l / 2 \pi$, неравенство (32) всегда имеет место для таких $p$. Таким образом, установлен критерий единственности.

Теорема 1. Если существует решение задачи (2)-(6), то оно единственно только тогда, когда выполнены условия (18) при всех $k \in \mathbb{N}_{0}$.

3. Построение решения задачи. Решение поставленной задачи (2)-(6) при выполнении условий (18) будем искать формально в виде суммы ряда

$$
u(x, y)=\frac{1}{\sqrt{l}} u_{0}(y)+\sqrt{\frac{2}{l}} \sum_{k=1}^{+\infty} u_{k}(y) \cos \gamma_{k} x+v_{k}(y) \sin \gamma_{k} x
$$

где коэффициенты $u_{0}(y), u_{k}(y)$ и $v_{k}(y)$ определяются соответственно формулами $(25),(21)$ и $(27)$. Поскольку $\Delta(k)$ входит в знаменатель коэффициентов ряда $(33)$ и, как показано выше, выражение $\Delta(k)$ имеет относительно $\widetilde{\alpha}$ счётное множество нулей (31), то при $\widetilde{\alpha}$, близких к корням уравнения (30), выражение $\Delta(k)$ может стать достаточно малым, т.е. возникает проблема «малых 
знаменателей» $[5,10,11]$. Следовательно, для обоснования существования решения задачи надо показать существование положительных чисел $\alpha, \beta, l$, $b$ и $\lambda$, при которых выражение $\Delta(k)$ отделено от нуля с соответствующей асимптотикой.

Лемма 1. Если $\tilde{\alpha}=p / q, p, q \in \mathbb{N},(q, 4)=1,(p, q)=1$, то существуют положительные постоянные $C_{0} u k_{0}\left(k_{0} \in \mathbb{N}\right)$, вообще говоря, зависящие от $\alpha, l, b$ и $\lambda$, такие, что при всех $k>k_{0}$ справедлива оченка

$$
|\Delta(k)| \geqslant C_{0} e^{\gamma_{k} \beta}>0
$$

Доказательство. При $b \neq 0$ выражение $\widetilde{\gamma}_{k}$, которое зависит от $l b$, при условии

$$
\frac{l b}{2 \pi k} \leqslant \frac{l b}{2 \pi}<1 \quad \text { или } \quad k>k_{1}=\frac{l b}{2 \pi}
$$

можно представить в виде

$$
\widetilde{\gamma}_{k}=\left(1+\left(\frac{l b}{2 \pi k}\right)^{2}\right)^{1 / 2}=1+r_{k}
$$

при этом для остатка $r_{k}$ справедлива оценка

$$
\frac{3}{8}\left(\frac{l b}{2 \pi k}\right)^{2}<r_{k}<\frac{1}{2}\left(\frac{l b}{2 \pi k}\right)^{2}
$$

Пусть $\alpha=p / q \in \mathbb{Q}$. В этом случае разделим $2 k p$ на $q$ с остатком:

$$
2 k p=s q+r
$$

Здесь $s, r \in \mathbb{N}_{0}, 0 \leqslant r<q$. Тогда выражение $\Delta(k)$ с учетом (30) и равенства (35) примет вид

$$
\Delta(k)=\sqrt{\operatorname{ch} 2 \gamma_{k} \beta}(-1)^{s+1} \sin \left(\frac{\pi r}{q}+\widetilde{\alpha} \widetilde{r}_{k}-\theta_{k}\right)+\frac{\lambda}{\gamma_{k}} \operatorname{sh} \gamma_{k} \beta, \quad \widetilde{r}_{k}=2 \pi k r_{k} .
$$

Поскольку $\theta_{k} \rightarrow \pi / 4$ при $k \rightarrow+\infty$ и последовательность $r_{k} \rightarrow 0$ в силу оценки (36), существует натуральное число $k_{2}$, такое, что при всех $k>k_{2}$

$$
|\delta(k)|=\left|\sin \left(\frac{\pi r}{q}+\widetilde{\alpha} \widetilde{r}_{k}-\theta_{k}\right)\right| \geqslant \frac{1}{2}\left|\sin \pi\left(\frac{r}{q}-\frac{1}{4}\right)\right|=C_{1}>0 .
$$

Тогда из (37) на основании оценки (38) имеем

$$
\begin{aligned}
& |\Delta(k)|=\sqrt{\operatorname{ch} 2 \gamma_{k} \beta}\left|(-1)^{s+1} \sin \left(\frac{\pi r}{q}+\widetilde{\alpha} \widetilde{r}_{k}-\theta_{k}\right)+\frac{\lambda}{\gamma_{k}} \frac{\operatorname{sh} \gamma_{k} \beta}{\sqrt{\operatorname{ch} 2 \gamma_{k} \beta}}\right|> \\
& >\frac{e^{\gamma_{k} \beta}}{\sqrt{2}}\left(|\delta(k)|-\frac{|\lambda|}{\gamma_{k}} \frac{1}{\sqrt{2}}\right)>\frac{e^{\gamma_{k} \beta}}{\sqrt{2}}\left(C_{1}-\frac{|\lambda|}{\mu_{k} \sqrt{2}}\right)=\frac{e^{\gamma_{k}}}{\sqrt{2}}\left(C_{1}-\frac{|\lambda| l}{2 \sqrt{2} \pi k}\right) .
\end{aligned}
$$


Отсюда видно, что существует число $k_{3} \in \mathbb{N}$, такое, что при всех

$$
k>k_{3}=\frac{|\lambda| l}{\sqrt{2} \pi C_{1}}
$$

справедливо неравенство

$$
\frac{|\lambda| l}{2 \pi \sqrt{2} k} \leqslant \frac{C_{1}}{2} .
$$

Тогда из (39) следует требуемая оценка (34) при всех $k>k_{0}=\max \left\{k_{1}, k_{2}, k_{3}\right\}$.

ЛЕмма 2. Пусть выполнены условия леммы 1. Тогда при всех $k>k_{0} u$ любом $y \in[-\alpha, \beta]$ справедливы оченки

$$
\begin{array}{cc}
\left|u_{k}(y)\right| \leqslant M_{1}\left(\left|\varphi_{k}\right|+\left|\psi_{k}\right|\right), & \left|u_{k}^{\prime}(y)\right| \leqslant M_{2} k\left(\left|\varphi_{k}\right|+\left|\psi_{k}\right|\right), \\
\left|v_{k}(y)\right| \leqslant M_{1}\left(\left|\tilde{\varphi}_{k}\right|+\left|\tilde{\psi}_{k}\right|\right), & \left|v_{k}^{\prime}(y)\right| \leqslant M_{2} k\left(\left|\tilde{\varphi}_{k}\right|+\left|\tilde{\psi}_{k}\right|\right), \\
\left|u_{k}^{\prime \prime}(y)\right| \leqslant M_{3} k^{2}\left(\left|\varphi_{k}\right|+\left|\psi_{k}\right|\right), & \left|v_{k}^{\prime \prime}(y)\right| \leqslant M_{3} k^{2}\left(\left|\tilde{\varphi}_{k}\right|+\left|\tilde{\psi}_{k}\right|\right),
\end{array}
$$

где $M_{i}$ - здесъ и далее положительные постоянные.

Доказательство. Справедливость приведённых в лемме 2 оценок непосредственно следует из формул (21)-(24) и (27) в силу оценки (34).

В силу леммы 2 ряд (33) и его производные первого порядка в замкнутой области $\bar{D}$ и производные второго порядка соответственно в областях $\bar{D}_{+}$и $\bar{D}_{-}$мажорируются числовым рядом

$$
M_{4} \sum_{k=k_{0}+1}^{+\infty} k^{2}\left(\left|\varphi_{k}\right|+\left|\psi_{k}\right|+\left|\tilde{\varphi}_{k}\right|+\left|\tilde{\psi}_{k}\right|\right) .
$$

Из теории рядов Фурье известно, что если $\varphi(x), \psi(x) \in C^{3}[0, l]$ и $\varphi^{(i)}(0)=$ $=\varphi^{(i)}(l), \psi^{(i)}(0)=\psi^{(i)}(l), i=0,1,2$, то ряд (40) оценивается сходящимся числовым рядом

$$
M_{5} \sum_{k=k_{0}+1}^{+\infty} \frac{1}{k}\left(\left|\varphi_{k}^{(3)}\right|+\left|\psi_{k}^{(3)}\right|+\left|\tilde{\varphi}_{k}^{(3)}\right|+\left|\tilde{\psi}_{k}^{(3)}\right|\right),
$$

где

$$
\begin{aligned}
\varphi_{k}^{(3)}= & \sqrt{\frac{2}{l}} \int_{0}^{1} \varphi^{(3)}(x) \sin \mu_{k} x d x, \quad \psi_{k}^{(3)}=\sqrt{\frac{2}{l}} \int_{0}^{1} \psi^{(3)}(x) \sin \mu_{k} x d x, \\
\widetilde{\varphi}_{k}^{(3)}= & \sqrt{\frac{2}{l}} \int_{0}^{1} \widetilde{\varphi}^{(3)}(x) \cos \mu_{k} x d x, \quad \widetilde{\psi}_{k}^{(3)}=\sqrt{\frac{2}{l}} \int_{0}^{1} \widetilde{\psi}^{(3)}(x) \cos \mu_{k} x d x, \\
& \sum_{k=1}^{+\infty}\left|\varphi_{k}^{(3)}\right|^{2} \leqslant\left\|\varphi^{(3)}(x)\right\|_{L_{2}}^{2}, \quad \sum_{k=1}^{+\infty}\left|\psi_{k}^{(3)}\right|^{2} \leqslant\left\|\psi^{(3)}(x)\right\|_{L_{2}}^{2}, \\
& \sum_{k=1}^{+\infty}\left|\widetilde{\varphi}_{k}^{(3)}\right|^{2} \leqslant\left\|\varphi^{(3)}(x)\right\|_{L_{2}}^{2}, \quad \sum_{k=1}^{+\infty}\left|\widetilde{\psi}_{k}^{(3)}\right|^{2} \leqslant\left\|\psi^{(3)}(x)\right\|_{L_{2}}^{2} .
\end{aligned}
$$


Если для указанных в лемме 1 чисел $\tilde{\alpha}$ выражение $\Delta(k) \neq 0$ при всех $k=\overline{0, k_{0}}$, то из сходимости ряда $(41)$ в силу признака Вейерштрасса сумма ряда (33) удовлетворяет условиям (2) и (3) при $F(x, y) \equiv 0$.

Если для указанных в лемме 1 чисел $\tilde{\alpha}$ при некоторых $k=k_{1}, k_{2}, \ldots, k_{p} \leqslant$ $k_{0}$, где $0 \leqslant k_{1} \leqslant k_{2}<\ldots<k_{p}, k_{i}, i=\overline{1, p}$ и $p$ - заданные натуральные числа, выражение $\Delta(k)=0$, то для разрешимости задачи (2)-(6) необходимо и достаточно, чтобы выполнялись условия

$$
\begin{aligned}
& \varphi_{k} \gamma_{k} \cos \gamma_{k} \alpha-\psi_{k} \operatorname{sh} \gamma_{k} \beta=0 \\
& \widetilde{\varphi}_{k} \gamma_{k} \cos \gamma_{k} \alpha-\widetilde{\psi}_{k} \operatorname{sh} \gamma_{k} \beta=0, \quad k=k_{1}, k_{2}, \ldots, k_{p} .
\end{aligned}
$$

Тогда решение задачи определяется в виде суммы

$$
\begin{aligned}
u(x, y)=\frac{1}{\sqrt{l}} u_{0}(y)+\sqrt{\frac{2}{l}} & \left(\sum_{k=1}^{k_{1}-1}+\sum_{k=k_{1}+1}^{k_{2}-1}+\ldots+\sum_{k=k_{p-1}+1}^{k_{p}-1}+\sum_{k=k_{p+1}+1}^{+\infty}\right) \times \\
& \times\left(u_{k}(y) \cos \gamma_{k} x+v_{k}(y) \sin \gamma_{k} x\right)+\sum_{m} \widetilde{u}_{m}(x, y),
\end{aligned}
$$

где в последней сумме $m$ принимает значения $k_{1}, k_{2}, \ldots, k_{p}$, функции $\widetilde{u}_{m}(x, y)$ определяются следующей формулой:

$$
\widetilde{u}_{p}(x, y)=\widetilde{u}_{p}(y) \cos \gamma_{p} x+\widetilde{v}_{p}(y) \sin \gamma_{p} x
$$

Здесь

$$
\begin{aligned}
& \widetilde{u}_{p}(y)= \begin{cases}\varphi_{p} \frac{\operatorname{sh} \gamma_{p} y}{\operatorname{sh} \gamma_{p} \beta}+C_{p} \frac{\operatorname{sh} \gamma_{p}(\beta-y)}{\operatorname{sh} \gamma_{p} \beta}, & y>0, \\
\psi_{p} \frac{\sin \gamma_{p} y}{\gamma_{p} \cos \gamma_{p} \alpha}+C_{p} \frac{\gamma_{p} \cos \gamma_{p}(y+\alpha)+\lambda \sin \gamma_{p} y}{\gamma_{p} \cos \gamma_{p} \alpha}, & y<0,\end{cases} \\
& \widetilde{v}_{p}(y)= \begin{cases}\widetilde{\varphi}_{p} \frac{\operatorname{sh} \gamma_{p} y}{\operatorname{sh} \gamma_{p} \beta}+C_{p} \frac{\operatorname{sh} \gamma_{p}(\beta-y)}{\operatorname{sh} \gamma_{p} \beta}, & y>0, \\
\tilde{\psi}_{p} \frac{\sin \gamma_{p} y}{\gamma_{p} \cos \gamma_{p} \alpha}+C_{p} \frac{\gamma_{p} \cos \gamma_{p}(y+\alpha)+\lambda \sin \gamma_{p} y}{\gamma_{p} \cos \gamma_{p} \alpha}, & y<0,\end{cases}
\end{aligned}
$$

а $C_{p}$ - произвольная постоянная.

Отметим, что равенства (44)-(46) составлены с учетом ненулевых решений (28) и (29) однородной задачи.

Таким образом, доказано следующее утверждение.

Теорема 2. Пусть выполнены условия леммы 1 (следовательно, выполнена оценка (34) при всех $\left.k>k_{0}\right)$ и $\varphi(x), \psi(x) \in C^{3}[0, l], \varphi^{(i)}(0)=\varphi^{(i)}(l)$, $\psi^{(i)}(0)=\psi^{(i)}(l), i=0,1,2$. Eсли при $\Delta(k) \neq 0$ nри всех $k=\overline{0, k_{0}}$, то существует единственное решение задачи (2)-(6) и это решение определяется рядом (33); если $\Delta(k)=0$ при некоторых $k=k_{1}, k_{2}, \ldots, k_{p} \leqslant k_{0}$, то задача (2)-(6) разрешима только тогда, когда выполнены условия (42) и решение в этом случае определяется рядом (43). 
4. Устойчивость решения задачи. Рассмотрим известные нормы

$$
\begin{gathered}
\|u\|_{L_{2}[0,1]}=\|u\|_{L_{2}}=\left(\int_{0}^{l}|u(x, y)|^{2} d x\right)^{1 / 2}, \\
\|u(x, y)\|_{C(\bar{D})}=\max _{\bar{D}}|u(x, y)| .
\end{gathered}
$$

Теорема 3. Пусть выполнены условия теоремы 2 и $\Delta(k) \neq 0$ при $k=$ $=\overline{0, k_{0}}$. Тогда для решения (33) задачи (2)-(6) имеют место следующие оцен$\kappa u$ :

$$
\begin{gathered}
\|u(x, y)\|_{L_{2}} \leqslant M_{6}\left(\|\varphi(x)\|_{L_{2}}+\|\psi(x)\|_{L_{2}}\right) \\
\|u(x, y)\|_{C(\bar{D})} \leqslant M_{7}\left(\|\varphi(x)\|_{L_{2}}+\|\psi(x)\|_{L_{2}}+\left\|\varphi^{\prime}(x)\right\|_{L_{2}}+\left\|\psi^{\prime}(x)\right\|_{L_{2}}\right),
\end{gathered}
$$

где постоянные $M_{6}$ и $M_{7}$ не зависят от функиий $\varphi(x)$ и $\psi(x)$.

Д о каз а тель ст в о проводится аналогично работе [5].

Благодарности. Работа выполнена при поддержке Российского фонда фундаментальных исследований (проект № 16-31-00421-мол_а).

\section{ORCID}

Виолетта Александровна Гущина: http://orcid.org/0000-0002-2801-3175

\section{БИБЛИОГРАФИЧЕСКИЙ СПИСОК}

1. Dezin A. A. On the solvable extensions of partial differential operators / Outlines Joint Sympos. Partial Differential Equations (Novosibirsk, 1963). Moscow: Acad. Sci. USSR Siberian Branch, 1963. pp. 65-66.

2. Дезин А. А. Операторы с первой производной по "времени" и нелокальные граничные условия // Изв. АН СССР. Сер. матем., 1967. Т. 31, №1. С. 61-86.

3. Нахушева 3. А. Об одной нелокальной задаче А. А. Дезина для уравнения Лаврентьева-Бицадзе // Дифферени. уравнения, 2009. Т. 45, № 8. С. 1199-2003.

4. Нахушева 3. А. Нелокальные краевые задачи для основных и смешанных типов дифференциальных уравнений, 2011. 196 с.

5. Сабитов К. Б., Новикова В. А. Нелокальная задача А. А. Дезина для уравнения Лаврентьева-Бицадзе // Изв. вузов. Матем., 2016. №6 (в печати).

6. Франкль Ф. И. Обтекание профилей потоком дозвуковой скорости со сверхзвуковой зоной, оканчивающейся прямым скачком уплотнения // ПМM, 1956. Т. 20, № 2. С. 196202.

7. Жегалов В. И. Краевая задача для уравнения смешанного типа с граничными условиями на обеих характеристиках и с разрывами на переходной линии / Краевые задачи теории аналитических функиий / Учен. зап. Казан. ун-та., Т. 122. Казань: Изд-во Казанского ун-та, 1962. С. 3-16.

8. Нахушев А. М. О некоторых краевых задачах для гиперболических уравнений и уравнений смешанного типа // Дифферени. уравнения, 1969. Т. 5, № 1. С. 44-59.

9. Сабитов К. Б. Задача Дирихле для уравнения смешанного типа // ДАН, 2007. Т. 413, № 1. C. $23-26$.

10. Сабитов К. Б., Сидоренко О. Г. Задача с условиями периодичности для вырождающегося уравнения смешанного типа // Дифферени. уравнения, 2010. Т. 46, № 1. С. 105-113.

11. Арнольд В. И. Малые знаменатели и проблемы устойчивости движения в классической и небесной механике // УМН, 1963. Т. 18, №6(114). С. 91-192.

Поступила в редакцию $07 / \mathrm{I} / 2016$; в окончательном варианте - 13/II/2016; принята в печать $-26 /$ II $/ 2016$. 
Vestn. Samar. Gos. Techn. Un-ta. Ser. Fiz.-mat. nauki

[J. Samara State Tech. Univ., Ser. Phys. \& Math. Sci.], 2016, vol. 20, no. 1, pp. 22-32

ISSN: 2310-7081 (online), 1991-8615 (print)

doi: http://dx.doi.org/10.14498/vsgtu1470

MSC: 35M12

\title{
THE NONLOCAL A. A. DESIN'S PROBLEM FOR AN EQUATION OF MIXED ELLIPTIC-HYPERBOLIC TYPE
}

\section{A. Gushchina}

Samara State University of Social and Humanities,

65/67, M. Gorky st., Samara, 443099, Russian Federation.

\begin{abstract}
In this paper for the equation of mixed elliptic-hyperbolic type in rectangular area the task with the conditions of periodicity and the nonlocal problem of A. A. Desin was studied, the uniqueness criterion was set. The solution of the problem was constructed as a sum of orthogonal series in eigenfunctions of the corresponding one-dimensional spectral problem. The problem of small denominators arises in justifying the convergence of the series. Therefore the evaluation on the separation from zero of small denominators with the corresponding asymptotics was established. This assessment allowed under certain conditions relative to the set objectives and functions to prove convergence of the constructed series in the class of regular solutions and the stability of the solution.
\end{abstract}

Keywords: equation of mixed type nonlocal problem, uniqueness criterion, the existence, series, small denominators, stability.

Acknowledgments. This work was supported by the Russian Foundation for Basic Research (project no. 16-31-00421-mol_a).

\section{ORCID}

Violetta A. Gushchina: http://orcid.org/0000-0002-2801-3175

\section{REFERENCES}

1. Dezin A. A. On the solvable extensions of partial differential operators, Outlines Joint Sympos. Partial Differential Equations (Novosibirsk, 1963). Moscow, Acad. Sci. USSR Siberian Branch, 1963, pp. 65-66.

2. Dezin A. A. Operators involving a first derivative with respect to time and nonlocal boundary conditions, Math. USSR-Izv., 1967, vol.1, no.1, pp. 57-79. doi:10.1070/ IM1967v001n01ABEH000547.

(C) 2016 Samara State Technical University.

Please cite this article in press as:

Gushchina V. A. The nonlocal A. A. Desin's problem for an equation of mixed elliptichyperbolic type, Vestn. Samar. Gos. Tekhn. Univ., Ser. Fiz.-Mat. Nauki [J. Samara State Tech. Univ., Ser. Phys. \& Math. Sci.], 2016, vol. 20, no. 1, pp. 22-32. doi: 10.14498/vsgtu1470. (In Russian)

\section{Author Details:}

Violetta A. Gushchina (violetta.novikova.1991@mail.ru), Postgraduate Student, Dept. of Physics, Mathematics and Methods of Teaching. 
3. Nakhusheva Z. A. On a nonlocal problem of A. A. Dezin for the Lavrent'evBitsadze equation, Differ. Equ., 2009, vol.45, no.8, pp. 1223-1228. doi: 10.1134/ S0012266109080151.

4. Nakhusheva Z. A. Nelokal'nye kraevye zadachi dlia osnovnykh i smeshannykh tipov differentsial'nykh uravnenii [Nonlocal boundary value problems for the main and mixed types of differential equations], 2011, 196 pp. (In Russian)

5. Sabitov K. B., Novikova V. A. Nonlocal A. A. Dezin's problem for Lavrent'ev-Bitsadze equation, Russian Mathematics (Izvestiya VUZ. Matematika), 2016, vol.60, no.6 (to appear).

6. Frankl' F. I. Subsonic flow about a profile with a supersonic zone, Prikl. Mat. Meh., 1956, vol. 20, no. 196-202 (In Russian).

7. Zhegalov V. I. A boundary-value problem for an equation of mixed type with boundary conditions on both characteristics and with discontinuities on the transition curve, Boundary value problems in the theory of analytic functions, Uchenye Zapiski Kazanskogo Universiteta, 122. Kazan, Kazan University, 1962, pp. 3-16 (In Russian).

8. Nakhushev A. M. On Some Boundary Value Problems for Hyperbolic Equations and Equations of Mixed Type, Differ. Uravn., 1969, vol. 5, no. 1, pp. 44-59 (In Russian).

9. Sabitov K. B. Dirichlet problem for mixed-type equations in a rectangular domain, Dokl. Math., 2007, vol.75, no. 2, pp. 193-196. doi: 10.1134/S1064562407020056.

10. Sabitov K. B., Sidorenko O. G. Problem with periodicity conditions for a degenerating equation of mixed type, Differ. Equ., 2010, vol.46, no.1, pp. 108-116. doi:10.1134/ S0012266110010118.

11. Arnol'd V. I. Small Denominators And Problems Of Stability Of Motion In Classical And Celestial Mechanics, Russian Math. Surveys, 1963, vol.18, no. 6, pp. 85-191. doi: 10.1070/ RM1963v018n06ABEH001143.

Received 07/I/2016;

received in revised form $13 / \mathrm{II} / 2016$;

accepted $26 / \mathrm{II} / 2016$. 